\title{
Tamper Evident Plastic Container Closure
}

National Cancer Institute

\section{Source}

National Cancer Institute. Tamper Evident Plastic Container Closure. NCI Thesaurus.

Code C96118.

A closure that shows the package has been opened and the product has been exposed to the outside environment. 